Note

\title{
Isolation and Properties of Paecilomyces sp. No. 5 Capable of Degrading High Concentrations of Formaldehyde
}

\author{
MASAYOSHI IWAHARA, RYOHEI FUKUDA, KEI NAKAHARA, KENJI SUZUKI, \\ TOSHIATSU TANAKA, AND YOSHIYUKI NOMURA*
}

Department of Applied Microbial Technology, Faculty of Engineering,

Sojo University, Ikeda 4-22-1, Kumamoto City 860-0082, Japan

Received 16 July 2001/Accepted 16 October 2001

\begin{abstract}
Six strains of fungi that degrade formaldehyde were isolated from the soil. Strain no. 5 degraded formaldehyde concentrations as high as $\mathbf{2 . 0 \%}$ within $\mathbf{2 0}$ days. Colonial and morphological properties indicated that strain no. 5 belongs to the genus Paecilomyces. This strain proliferated in medium containing $1.5 \%$ formaldehyde as the sole carbon source. The upper concentration limit of formaldehyde that permitted fungal growth was $2.4 \%$ and strain no. 5 degraded that concentration of formaldehyde completely within $\mathbf{5 5}$ days.
\end{abstract}

Key words : Biodegradation/Fungus degradation of formaldehyde/Formaldehyde assimilation/ Paecilomyces sp.

As formaldehyde can act as a disinfectant at concentrations as low as $0.1 \%$, it has been used for disinfecting and preserving experimental specimens. Moreover formaldehyde is a ubiquitous compound that is a product of biological (photooxidation of atmospheric hydrocarbons) (Levy, 1971; Zimmerman et al., 1978) and environmental sources (emissions from industrial products) (Ando, 1998). Formaldehyde is harmful to humans and is an environmental pollutant.

To resolve the problem of environmental pollution caused by formaldehyde, we searched for microorganisms that can degrade it even at high concentrations. Samples of soil collected in Kumamoto prefecture were incubated at $25^{\circ} \mathrm{C}$ in I-medium (see Table 1) containing $0.1 \%$ formaldehyde using test tubes on the reciprocal shaker (120 strokes per min). All reagents in this experiments were purchased from

TABLE 1. Composition of media.

\begin{tabular}{|c|c|c|c|c|c|c|c|}
\hline \multirow{2}{*}{ Media } & \multicolumn{7}{|c|}{ Components of media (\%) } \\
\hline & Glucose & Yeast extract D-3 & Malt extract & Polypeptone & Formaldehyde $^{a}$ & $\mathrm{CMC}^{b}$ & Agar \\
\hline I-medium & 1.0 & 0.3 & 0.3 & 0.5 & $0.1-1.5$ & & \\
\hline Medium for stock culture & 0.5 & 0.3 & 0.3 & 1.0 & 0.5 & & 1.5 \\
\hline Medium for seed culture & 0.5 & 0.3 & 0.3 & 1.0 & 0.3 & 3.0 & \\
\hline D-medium & 0.5 & 0.3 & 0.3 & 1.0 & $1.5-2.5$ & & \\
\hline
\end{tabular}

$\mathrm{pH}$ of media was adjusted at 7.0 .

${ }^{a}$ Formaldehyde solution (36-38\%, w/v, special grade) was used.

${ }^{b}$ Carboxymethyl cellulose sodium salt.

*Corresponding author. Tel: +81-96-326-3111, Fax : +81-

96-323-1330. 
Wako Pure Chemical Industries, Ltd., except for malt extract (Oriental Yeast Co., Ltd.). Microorganisms that thrived in this medium were transferred to Imedium containing $0.2 \%$ formaldehyde and incubated in a similar manner. Microorganisms were then transferred to the I-medium containing higher concentrations of formaldehyde (acclimatization). Finally, microorganisms that proliferated in medium containing $1.5 \%$ formaldehyde were plated on the medium for stock culture. After conidia formation, microorganisms were purified by being plated several times on the agar medium. In this way, we isolated six strains of microorganisms, numbered 2, 5, 7, 14, 16 and 105. All six strains degraded $1.5 \%$ formaldehyde and all were fungi. To examine the degradation activity of these isolates using D-medium containing 2.0\% formaldehyde, the stock culture of each isolate $(5 \mathrm{~mm}$ $\times 5 \mathrm{~mm}$, containing $(1.5 \pm 0.02) \times 10^{7}$ conidia) was inoculated into $100 \mathrm{ml}$ of medium for seed culture and incubated on a rotary shaker $(220 \mathrm{rpm})$ at $25^{\circ} \mathrm{C}$ for 5 days. Erlenmeyer flasks containing $100 \mathrm{ml}$ of Dmedium were inoculated with $1.5 \%(\mathrm{v} / \mathrm{V})$ seed culture and incubated on a rotary shaker at $25^{\circ} \mathrm{C}$. The concentration of formaldehyde was determined using the Formaldehyde-Test Wako kit. The time courses of formaldehyde degradation by these isolates are shown in Fig. 1. In this figure, measurement values of formaldehyde are sometimes higher than those of the earlier samples. This must be an experimental error (also the same in Fig. 3 and 4), because the probable error was $\pm 0.05 \%$ in formaldehyde determination kit. No formaldehyde was lost due to volatilization. After 12 days of incubation, strain no. 5 degraded formaldehyde rapidly, and after 20 days, this strain had completely degraded $2.0 \%$ formaldehyde. Strains no. 14

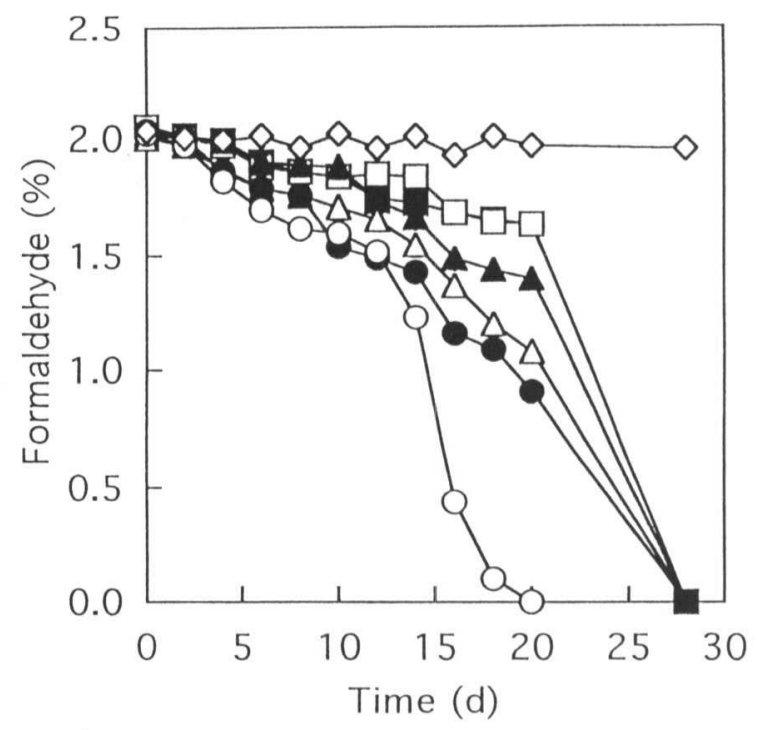

FIG. 1. Time courses of formaldehyde degradation by isolates. Fungal cells were incubated at $25^{\circ} \mathrm{C}$ on a rotary shaker in Erlenmeyer flasks containing $100 \mathrm{ml}$ of D-medium including $2.0 \%$ formaldehyde. Symbols: $\diamond$, without microbes; $\mathbf{\square}$, no. $2 ; \bigcirc$, no. $5 ; \square$, no. $7 ; \triangle$, no. $14 ; \bigcirc$, no. 16 ; $\boldsymbol{\Delta}$, no. 105 .

and no. 16 degraded about half the formaldehyde added within 20 days of incubation. After 28 days, all other strains also totally degraded $2.0 \%$ formaldehyde. Until now, most microorganisms reported to degrade formaldehyde have been bacteria (Ando et al., 1979; Barber and Donohue, 1998; Ras et al., 1995). To our knowledge, only a few reports describe fungi that can degrade formaldehyde (Sakaguchi et al., 1975) at concentrations below $0.2 \%$. We are the first to describe microorganisms that can degrade concen-
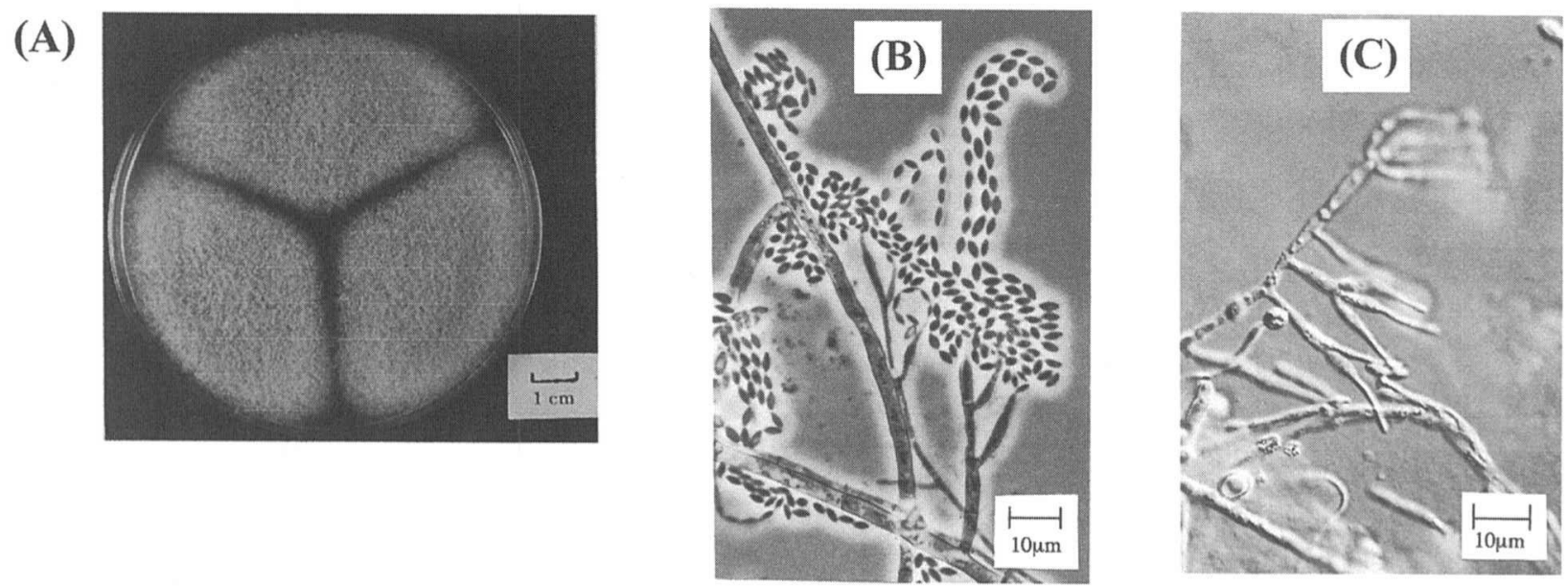

FIG. 2. Photographs of strain no. 5. (A): photograph of the plate culture of strain no. 5 using malt extract medium. Microscopy photographs of strain no. 5. (B): phase-contrast microscopy. (C) : differential interference-contrast microscopy. Magnifications in microscopy photographs are $\times 600$. Strain no. 5 was incubated at $25^{\circ} \mathrm{C}$ for 1 week using malt extract agar. 
trations of formaldehyde as high as $2.0 \%$. Colony observation indicated that the six strains belong to the same genus. It was considered that the same genus of fungus was obtained owing to acclimatization under high concentrations of formaldehyde. Among them, as strain no. 5 had a prominent ability to degrade formaldehyde, we decided to examine some properties of no. 5 .

A taxonomical study of strain no. 5 that degraded high concentrations of formaldehyde was performed at the National Collections of Industrial and Marine Bacteria (NCIMB), Japan Co., Ltd. (Shimizu, Shizuoka Prefecture). A photograph of a colony of strain no. 5 is shown in Fig. 2A. This strain proliferated quickly on malt extract agar and a colony with a $4.5 \mathrm{~cm}$ diameter was formed within 5 days. After one week of incubation, the colony surface was yellowish brown. Photographs from phase-contrast and differential interference-contrast microscopy are shown in Fig. 2B and $2 \mathrm{C}$, respectively. Phialides were long and thin $(2.5-3.0 \times 14-16 \mu \mathrm{m})$ and were monoverticillus, biverticillus or irregular branching. Chlamydospores (3.0-6.0 $\times 4.0-8.0 \mu \mathrm{m})$ were formed. Conidia were elliptical $(2.5-3.0 \times 3.5-4.5 \mu \mathrm{m})$ and with a smooth surface. Teleomorphs were absent. According to the method of Samson (1974), strain no. 5 belonged to the genus Paecilomyces. Therefore, the strain was identified as Paecilomyces sp. no. 5.

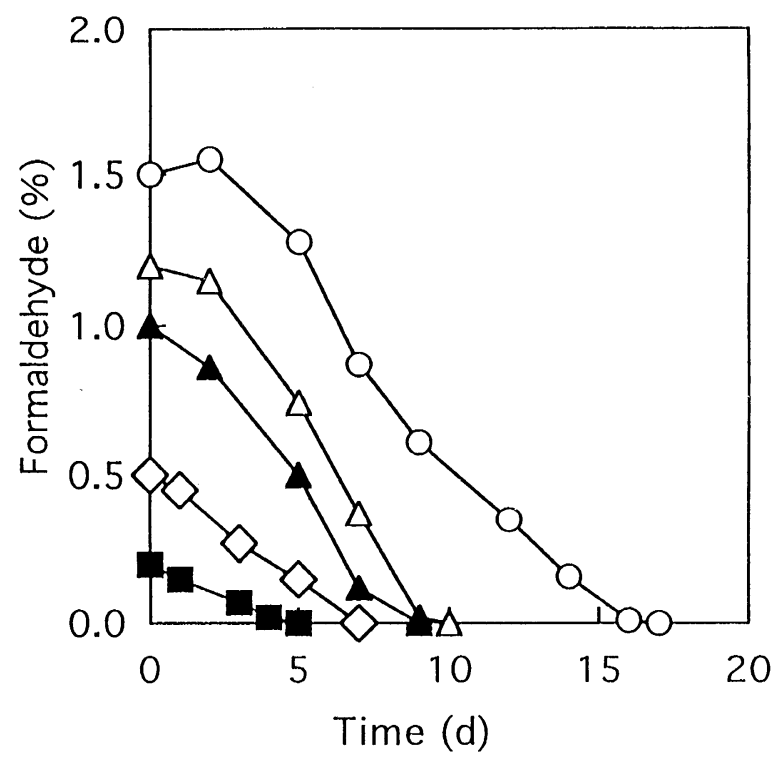

FIG.3. Assimilation of formaldehyde by Paecilomyces $\mathrm{sp}$. no. 5. Cells were incubated at $25^{\circ} \mathrm{C}$ on a rotary shaker in Erlenmeyer flasks containing $100 \mathrm{ml}$ of medium as described by Sakaguchi et al. (see text). Various concentrations of formaldehyde were added as the sole carbon source. Symbols: $\square, 0.2 \% ; \square, 0.5 \% ; \boldsymbol{\Delta}, 1.0 \% ; \triangle, 1.2 \%$; $0,1.5 \%$ formaldehyde.

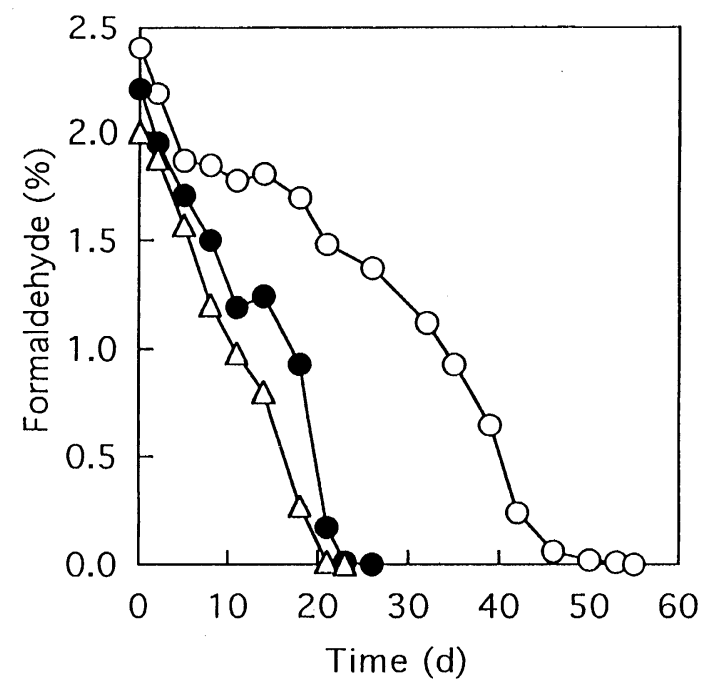

FIG.4. The upper concentration limit of formaldehyde degradation by Paecilomyces sp. no. 5. Incubation conditions were the same as those described in the legends of Figure 1 except for the concentration of formaldehyde. The concentrations here are $2.0 \%(\triangle), 2.2 \%(\bigcirc), 2.4 \%(\bigcirc)$.

Sakaguchi et al.(1975) reported that Paecilomyces varioti can assimilate formaldehyde. The upper concentration limit of formaldehyde which permitted the growth of $P$. varioti was $0.2 \%$. To investigate the assimilation of formaldehyde by Paecilomyces sp. no. 5, we used the medium described by Sakaguchi et al., which contained $0.15 \%\left(\mathrm{NH}_{4}\right)_{2} \mathrm{HPO}_{4}, 0.1 \% \mathrm{KH}_{2} \mathrm{PO}_{4}$, $0.1 \% \mathrm{KNO}_{3}, 0.05 \% \mathrm{MgSO}_{4} \cdot 7 \mathrm{H}_{2} \mathrm{O}, 0.1 \%$ yeast extract and formaldehyde ( $\mathrm{pH}$ 7.0). As described above, we inoculated the seed culture of strain no. 5 into Erlenmeyer flasks with $100 \mathrm{ml}$ of medium containing various concentrations of formaldehyde (0.2$1.5 \%)$ as the sole carbon source and incubated them on a rotary shaker. Strain no. 5 proliferated even in a medium containing $1.5 \%$ formaldehyde (Fig. 3 ), and consumed $1.5 \%$ formaldehyde within 17 days and the yield of mycelium as dry matter was $0.31 \mathrm{mg} / \mathrm{ml}$.

We also examined the upper concentration limit of formaldehyde degradation by strain no. 5 in Dmedium. A seed culture of strain no. 5 was inoculated into Erlenmeyer flasks with $100 \mathrm{ml}$ of D-medium containing various concentrations of formaldehyde (2.0$2.5 \%$ ) and the flasks were incubated on a rotary shaker. The time course of degradation by strain no. 5 is shown in Fig. 4. Strain no. 5 proliferated in Dmedium containing $2.4 \%$ formaldehyde. This strain degraded $2.0 \%$ and $2.2 \%$ of formaldehyde linearly within 20-25 days, and needed 55 days to degrade $2.4 \%$ of formaldehyde. This strain could not grow in D-medium containing $2.5 \%$ of formaldehyde owing to the toxicity of formaldehyde.

Thus, Paecilomyces sp. no. 5 can grow in medium 
containing concentrations of formaldehyde as high as $2.4 \%$; yeasts and bacteria can usually grow in only 0.01-0.04\% formaldehyde. Paecilomyces sp. no. 5 has prominent ability for formaldehyde degradation. Therefore, this strain will useful in the biological degradation of waste water containing formaldehyde.

\section{ACKNOWLEDGMENT}

The authors thank Toshiaki Kishikawa for his technical assistance.

\section{REFERENCES}

Ando, M. (1998) Indoor air pollution and chemical substances. 10th. Aliphatic hydrocarbons, esters, carboxyls, fats, ethers, terpenes, and others. 1. Formaldehyde, Shigen Kankyo Taisaku (in Japanese), 34, 53-60.

Ando, M., Yoshimoto, T., Ogushi, S., Rikitake, K., Shibata, S., and Tsuru, D. (1979) Formaldehyde dehydrogenase from Pseudomonas putida. Purification and some
Properties. J. Biochem., 85, 1165-1172.

Barber, R. D., and Donohue, T. J. (1998) Function of a glutathione-dependent formaldehyde dehydrogenase in Rhodobacter sphaeroides formaldehyde oxidation and assimilation. Biochemistry, 37, 530-537.

Levy, H. (1971) Normal atmosphere: Large radical and formaldehyde concentrations predicted. Science, 173, 141143.

Ras, J., Van, Ophem, P. W., Reijnders, W. N., Van, Spanning, R. J., and Duine, J. A. (1995) Isolation, sequencing, and mutagenesis of the gene encoding NAD-and glutathionedependent formaldehyde dehydrogenase (GD-FALDH) from Paracoccus denitrificans, in which GD-FALDH is essential for methylotrophic growth. J. Bacteriol., 177, 247251.

Sakaguchi, K., Kurane, R., and Murata, M. (1975) Assimilation of formaldehyde and other C1-compounds by Gliocladium deliquescens and Paecilomyces varioti. Agric. Biol. Chem., 39, 1695-1702.

Samson, R.A. (1974) Paecilomyces and some allied hyphomycetes. In Studies in Mycology No. 6. p 116, Centraalbureau voor Schimmelcultures, Baarn, Netherlands. 